University of Nebraska - Lincoln

DigitalCommons@University of Nebraska - Lincoln

Mammalogy Papers: University of Nebraska

State Museum

Museum, University of Nebraska State

$11-7-2007$

\title{
The Trade-Off between Tooth Strength and Tooth Penetration: Predicting Optimal Shape of Canine Teeth
}

\author{
Patricia W. Freeman \\ University of Nebraska-Lincoln, pfreeman1@unl.edu \\ Cliff A. Lemen \\ University of Nebraska-Lincoln, clemen2@unl.edu
}

Follow this and additional works at: https://digitalcommons.unl.edu/museummammalogy

Part of the Zoology Commons

Freeman, Patricia W. and Lemen, Cliff A., "The Trade-Off between Tooth Strength and Tooth Penetration: Predicting Optimal Shape of Canine Teeth" (2007). Mammalogy Papers: University of Nebraska State Museum. 33.

https://digitalcommons.unl.edu/museummammalogy/33

This Article is brought to you for free and open access by the Museum, University of Nebraska State at DigitalCommons@University of Nebraska - Lincoln. It has been accepted for inclusion in Mammalogy Papers: University of Nebraska State Museum by an authorized administrator of DigitalCommons@University of Nebraska Lincoln. 


\title{
The trade-off between tooth strength and tooth penetration: Predicting optimal shape of canine teeth
}

\author{
P. W. Freeman and C. A. Lemen \\ School of Natural Resources and University of Nebraska State Museum, University of Nebraska-Lincoln, Lincoln, NE, USA
}

\author{
Received December 20, 2006; accepted \\ March 6, 2007. \\ Corresponding author: Patricia W. Freeman, \\ School of Natural Resources, University \\ of Nebraska-Lincoln, Lincoln, Nebraska, \\ USA. Email: pfreeman1@unl.edu \\ Keywords: \\ optimal canine shape \\ tooth strength \\ tooth sharpness \\ tooth penetration \\ vertebrate teeth
}

\section{Introduction}

Can the shape of a canine tooth be predicted based on optimal design criteria? Alexander (1982) used an optimization approach to predict the size of the marrow cavity within mammalian long bones.He postulated that the size of themarrow cavity was an evolutionary trade-off between weight and strength. He showed that for a given bending strength, weight is minimized with a certain ratio of bone diameter to marrow diameter. This ratio closely matches real values. Here, we use this evolutionary trade-off approach to predict the shape of a canine tooth. There is an important difference in the bone/marrow and tooth systems. The pulp cavity is reduced to a tiny diameter with age in most mammals. This reduction maximizes the tooth's strength and weight. Evidently, the weight-strength trade-off is not a significant issue in the evolution of tooth design.

When considering the strength of an object, such as a canine tooth, both the material from which it is made and the shape it takes must be considered. Evidence from the field indicates that wild carnivores often break their canines (Van Valkenburgh, 1988). Even dentin, one of the strongest materials a mammal can make, is not strong enough to stand up to the rough usage these teeth face. The obvious question is why are the teeth not thicker and/or blunter to improve their strength? Strength is not the only issue to consider in tooth design; teeth must also penetrate prey. There is a compromise between a tooth's strength and the ease with which it will penetrate prey. The sharper the tooth, the easier it can penetrate, but the weaker it becomes.
Evans \& Sanson (1998) quantified the relationship between force of penetration and the sharpness of a tooth in a series of experiments puncturing insects. They constructed a series of artificial teeth that varied in sharpness and width and concluded that both the radius of curvature of the tip and size of tooth were important. We use a similar approach here, but we puncture mammalian hide and our artificial teeth broadly mimic felid upper canines. Our goal is to extend the basic approach of Evans \& Sanson (1998) by attempting to predict the optimum shape of a canine tooth. We experimentally quantify the trade-off between tooth weakness (based on beam theory, Popov, 1999) and force of penetration by using artificial teeth of known shape to puncture both fresh pig and deer hide. The results from these experiments allow us to estimate the relative importance of the fitness costs of tooth breakage and force of penetration and to predict the optimal aspect ratio (tooth height/base diameter) for a canine.

We made some important simplifying assumptions from the outset. One important choice we made was to restrict the shapes of the canines we modeled. Although canines are relatively simple in form compared with mammalian premolars and molars, there are important differences in the shapes of canines across mammalian groups. Therefore, we needed to restrict our investigation to a narrower range of diversity. Because we constructed artificial steel canines on a lathe, our artificial teeth are round in cross-section. In some carnivores, particularly canids, the upper canine is strongly oval, with the ratio of the major to minor axis being about 1.54 (Van Valkenburgh \& Ruff, 1987). Felids on the other hand, have ratios of 
about 1.30. Thus, we concentrated our attention on modeling felid canines for the practical limitation that our artificial teeth most closely resemble these teeth. The range of shapes of the artificial teeth were constructed to include the basic shape differences of canines found in extant species of cats.

We also assumed that an important function of a canine is to puncture the hide of prey or enemies. Here, we used the hides of two mammals (domestic pig and white-tailed deer) to measure this function. Of course, this ignores most of the diversity of mammalian skin across all mammals based on taxa and size, and many cats take non-mammalian prey as well. However, without this simplifying assumption the experimental design could quickly become intractable as more food types are included.

\section{Materials and methods}

\section{Quantifying tooth size and shape}

Three measures simplify understanding the size and shape of canines. We measured tooth height from the socket in the maxillary bone to the tip of the tooth. Second, the aspect ratio of the tooth is tooth height/the major diameter (anterior-posterior in most mammals, mesiodistal in humans) at the base of the tooth. And third is a function that describes the taper in diameter of a standardized tooth along its length. A standardized tooth is one that has been mathematically standardized to have a height and basal diameter of one. Fortunately, we found a simple function that can closely model real felid canines:

$$
D_{x}=L_{x}^{\varepsilon}
$$

where $D_{x}$ is the anterior-posterior diameter of the tooth at point $x$ and $L_{x}$ is the distance from the tooth's tip to point $x$ along the tooth's shank. Only one parameter is needed, $\varepsilon$, to describe the shape of a standardized tooth. We will refer to $\varepsilon$ as the taper parameter. The relationship between tooth shape and $\varepsilon$ is shown in Figure 1. In this paper, we analyze teeth with values of $\varepsilon$ that range from 1.0 (a straight-sided cone) to 0.33 (a blunt-tipped cone).

\section{Felid teeth}

Specimens were selected to have little or no wear on their teeth from the collection of the University of Nebraska State Museum. We used clouded leopard Neofelis nebulosa, cheetah Acinonyx jubatus, leopard Panthera pardus, tiger Panthera tigris, lion Panthera leo, puma Puma concolor, bobcat Lynx rufus, ocelot Felis pardalis, and house cat Felis catus. This sample of species represents a cross-section of extant species. We have included the largest species (tiger) and one of the smallest (house cat) ones. Also, the clouded leopard has the longest canine relative to the body size of any extant felid. To calculate $\varepsilon$ for teeth, we photographed lateral views of these felid upper canines and measured the anterior-posterior diameters of the canines along the shank. We standardized the data to a tooth height and maximal width of 1 and found the $\varepsilon$ that fit the data best with the least squares method (Figure 2).

\section{Weakness of teeth}

We determined tooth weakness using simple beam theory (Popov, 1999). Our approach here was to concentrate on tip weakness. Tip weakness of the tooth is defined as the bending

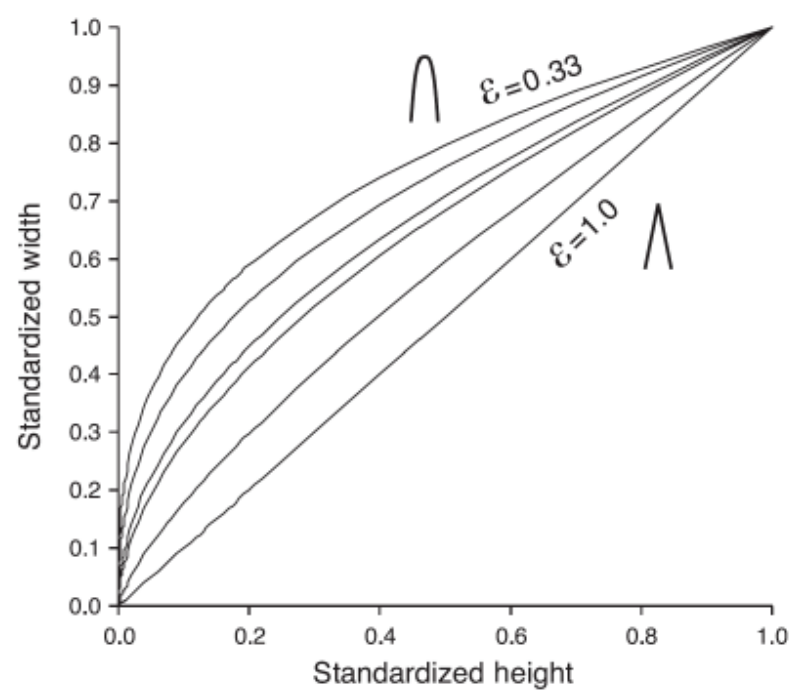

Figure 1 Relationship between standardized height and standardized width along the shank of a tooth based on equation (1). The curves correspond to different values of the taper parameter, $\varepsilon(1.0,0.75$, $0.55,0.5,0.4$ and 0.333 ).

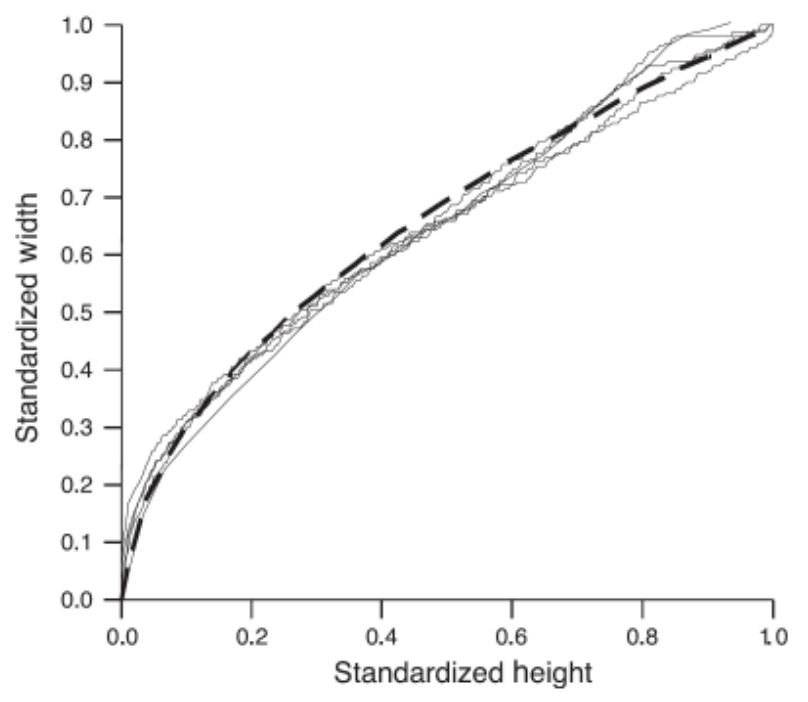

Figure 2 Relationship between standardized height and standardized width along the shank of a tooth for five species of cats (leopard Panthera pardus, puma Puma concolor, cheetah Acinonyx jubatus, clouded leopard Neofelis nebulosa, and tiger Panthera tigris) and line based on $\varepsilon=0.55$ from equation (1). 
stress near the tip of the tooth generated by a load at the tooth's tip. In our case, we calculated tip weakness at a point 5 and $20 \%$ from the tip. When a load is applied at the tip of a tooth, it produces a bending moment $M$ along the shank that is given by:

$$
M=L_{x} F
$$

$F$ is the concentrated force (load) applied at the tip, which for ease of calculation will be given as $1.0 \mathrm{~N}$. To calculate the stress at any point along the shank, we also need the moment of inertia, I, for the shank at point x. For a round cross-sectional beam, this is simply:

$$
I=\pi r^{4} / 4
$$

where $r$ is the radius of the shank at point $x$. Finally, the maximum bending stress produced, $s$, at point $x$ can be estimated as:

$$
\sigma=M r / I
$$

The amount of stress experienced at a point $5 \%$ from the tip and $20 \%$ from the tip is calculated for all teeth. Stress is our index of the weakness of a tooth because high stress occurs in weak cross-sections.

\section{Artificial teeth}

Artificial teeth were made on a computer-controlled Sherline lathe (Sherline Products Inc., 3235 Executive Ridge, Vista, CA, USA) from stainless steel. The height of all teeth was set at $11.9 \mathrm{~mm}$. In one series, artificial teeth varied only in $\varepsilon$, the taper parameter, and had the same height and width at base (Figure 3a). Teeth were produced with $\varepsilon$ values of $1.0,0.75$, $0.55,0.5,0.4$ and 0.333 . This series of teeth is referred to as the $\varepsilon$ series. All artificial teeth in the $\varepsilon$ series had the same aspect ratio of 2.5 (which is roughly consistent with felids). In addition, a set of artificial teeth was made with $\varepsilon=0.55$ (which is roughly consistent with felids) and aspect ratios of 3.0, 2.5, 2.4, 2.2, 2.0 and 1.876 (Figure 3b). This series of teeth is referred to as the aspect ratio series. Changes in aspect ratio were made by varying the width of the tooth's base. All teeth had the same height.

\section{Measuring force of penetration}

We measured force of penetration in newtons by mounting the artificial teeth like a drill bit in a drill press into an Inspec 2200, a uniaxial compression machine from Instron (Instron Corp. 825 University Ave. Norwood, MA, USA). We punctured both pig Sus scrofa domesticus hide (from pork bellies with about $25 \mathrm{~mm}$ of fat and meat under the skin) and fresh white-tailed deer Odocoileus virginianus hide (hide alone; all meat was removed) using six replicates in all cases. The hides were placed over a 30 -mm-diameter steel cylinder with a 10 $\mathrm{mm}$ hole bored into it to accommodate the artificial teeth during penetration. The hide was held by hand around the edge of the cylinder to prevent the hide from simply sliding down the hole. We measured the relationship between downward

movement of the tooth and the force of resistance to penetration with our Inspec machine (Figure 4); movement was set at a constant $1 \mathrm{~mm} \mathrm{~s}^{-1}$. Normally, the initial penetration of the hide is visible in these graphs (arrows in Figure 4).

\section{Results}

\section{Quantifying tooth size and shape}

Aspect ratios of extant felids are shown in Table 1. Analysis of several species shows that equation (1) does a remarkably good job of modeling the changes in diameter of the canine (Figure 2). This function did not model the shapes of canine teeth in canids or ursids nearly as well because of the rapid increase of the anterior-posterior diameter near the base of these oval canine teeth. The values of $\varepsilon$ found for cats are shown in Table 2. They average 0.55 and $\varepsilon$ is similar across these felids. The standard deviation is 0.0128 yielding a CV of $2.33 \%$.

The range of $\varepsilon$ values used in this study for artificial teeth (1.0-0.333) more than captures the range we found in felid canines (Table 2). When $\varepsilon=1.0$, a straight-sided cone is produced (Figure 3a). This tooth represents an extreme in sharpness and might be predicted as the optimum shape if ease of penetration were the only issue. With $\varepsilon=0.333$, a blunt tooth is formed with the interesting property that a load applied at the tip produces equal bending stresses along the shank of the tooth right down to the base. This design could be considered an extreme in favor of strength (in resistance to bending). Further blunting of the tip would produce a tooth more susceptible to breakage at the base than at the tip. Because of symmorphosis (Weibel, 2000), we would not expect the tip to be over-engineered compared with the rest of the structure.

(a)

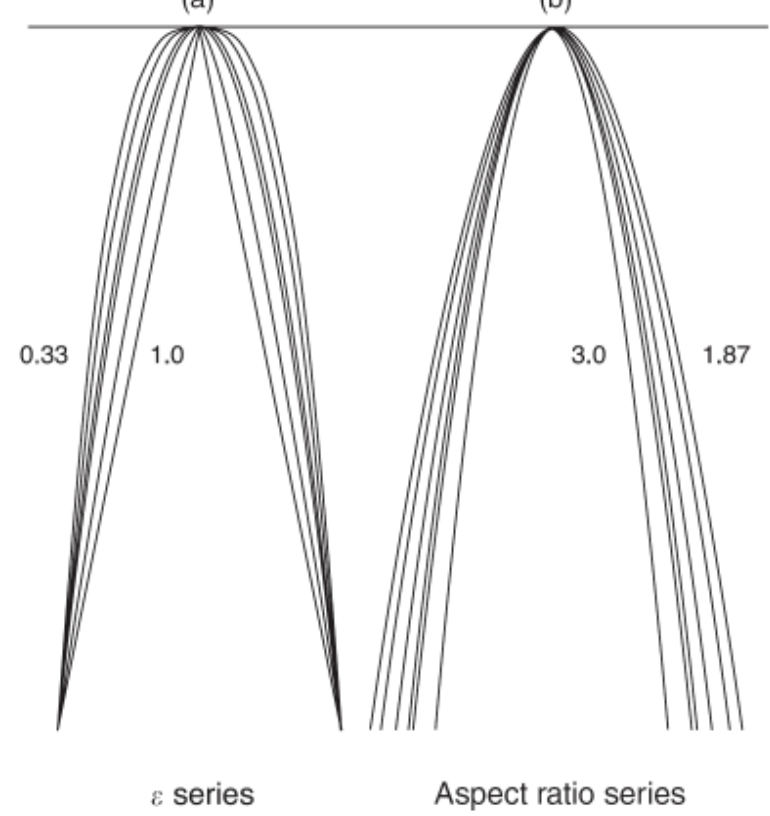

Figure 3 Drawings of the artificial teeth from both the $\varepsilon$ tooth series (a) and the aspect ratio series (b). 


\section{Weakness of teeth}

The weakness of the canine tip is strongly affected by both the taper parameter $\varepsilon$ (Figure 5a) and aspect ratio (Figure 5b). A higher value of $\varepsilon$ means a sharper tooth. A higher aspect ratio means that the tooth is narrower at its base than a tooth with a low aspect ratio. As might be expected, the straight-sided cone with $\varepsilon=1.0$ has the weakest tip and the most blunt tooth $(\varepsilon=0.333)$ has the strongest tip. The point at which tip weakness is measured along the length of the tooth has a strong impact on the relative weakness among teeth. If weakness is measured near the base of the tooth on our $\varepsilon$ series, all teeth have the same weakness because they all have the same diameter at the base of the tooth. The closer to the tip we measured weakness, the greater the differences among these teeth. Note in Figure 5a that there is less relative difference in weakness when it is measured at the $20 \%$ position than at the $5 \%$ position. Thus, measuring tip weakness farther from the tip will tend to favor sharp-tipped teeth because the sharp, hence
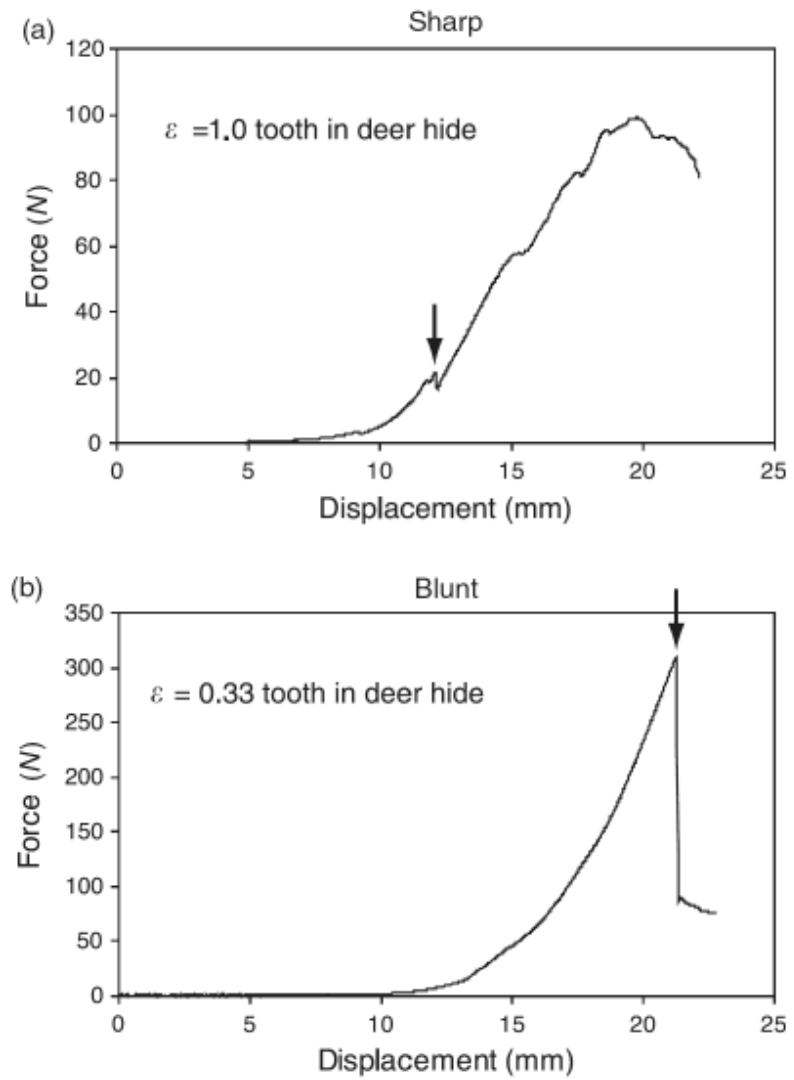

Figure 4 Relationship between downward displacement of the artificial tooth into deer Odocoileus virginianus hide and the force required for movement at $1 \mathrm{~mm} \mathrm{~s}^{-1}$. (a) Displacement/force curve for the sharp ( $\varepsilon$ series 1.0$)$ tooth. The arrow indicates that the deer hide is initially penetrated at a very low force (about $17 \mathrm{~N}$ ). However, this tiny prick in the hide must be widened by the rest of the tooth's shank to achieve full penetration. This requires considerably more force (about $100 \mathrm{~N}$ ). (b) Displacement/force curve for the blunt ( $\varepsilon$ series 0.333 ) tooth. The arrow represents the point of catastrophic failure of the hide when initial penetration takes place (about $300 \mathrm{~N}$ ).
Table 1 Aspect ratios (tooth height/maximal width at base) for some felids

\begin{tabular}{ll} 
Clouded leopard Neofelis nebulosa & 3.28 \\
Cheetah Acinonyx jubatus & 2.13 \\
Leopard Panthera pardus & 2.44 \\
Puma Puma concolor & 2.24 \\
Tiger Panthera tigris & 2.12 \\
Lion Panthera leo & 2.15 \\
Bobcat Lynx rufus & 2.42 \\
Ocelot Felis pardalis & 2.26 \\
House cat Felis catus & 2.41 \\
Mean & 2.39 \\
\hline
\end{tabular}

Table 2 Taper parameter, $\varepsilon$, for some felids

\begin{tabular}{ll}
\hline Clouded leopard Neofelis nebulosa & 0.57 \\
Cheetah Acinonyx jubatus & 0.55 \\
Leopard Panthera pardus & 0.53 \\
Puma Puma concolor & 0.54 \\
Tiger Panthera tigris & 0.55 \\
Ocelot Felis pardalis & 0.55 \\
Mean & 0.55 \\
\hline
\end{tabular}
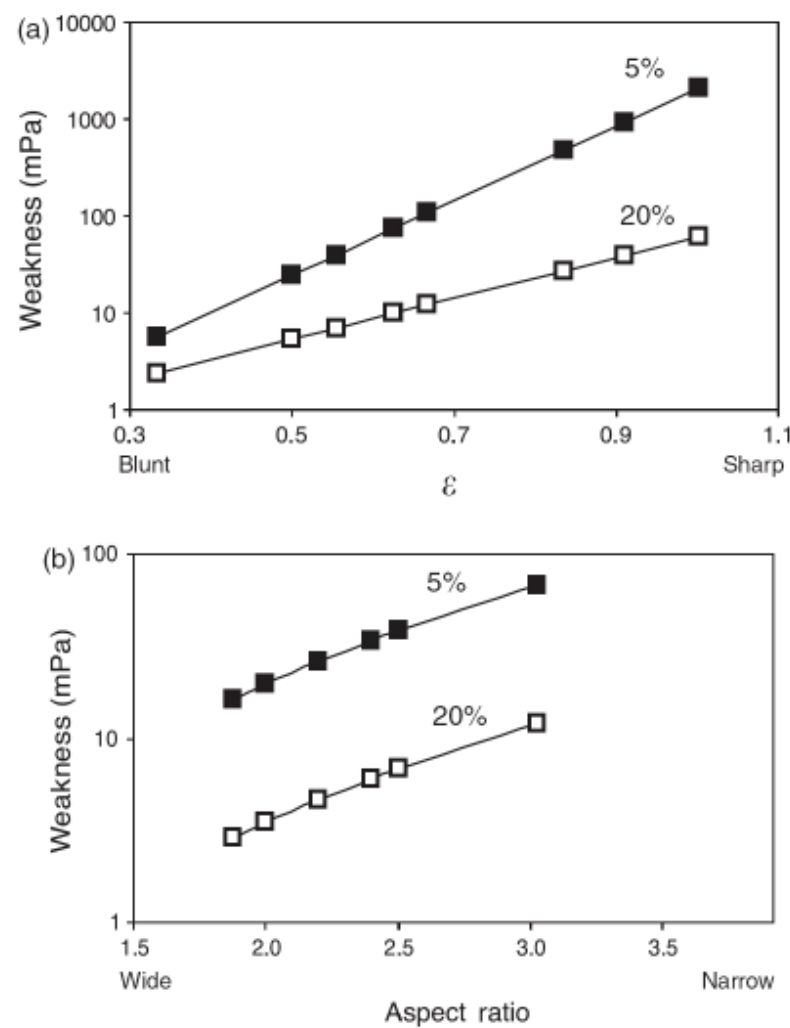

Figure $\mathbf{5}$ The effect of tooth shape on tooth weakness is shown. Weakness is calculated as a stress created by a point load at the tooth's tip. (a) Relationship of $\varepsilon$ and weakness measured at a point $5 \%$ from the tooth's tip (solid squares) and $20 \%$ from the tip (open squares). (b) Relationship of aspect ratio and weakness measured on a tooth with $\varepsilon=$ 0.55 . As before, weakness is measured at a point $5 \%$ from the tooth's tip (solid squares) and $20 \%$ from the tip (open squares). 
weak, tips of these teeth are not taken into account, only their relatively stronger shanks. However, in our analysis, results using the 5 and $20 \%$ weakness criteria are similar and did not alter our conclusions.

\section{Force of penetration}

The maximum force required to drive the $\varepsilon$ series teeth through hide to the base of the tooth is shown in Figure 6a. Not surprisingly, blunt teeth require a great deal more force to penetrate than sharp teeth (Figure 6a). However, for both deer and pig hide, the advantage to the sharpest tooth, the straight-sided cone, is small or non-existent compared with the tooth with $\varepsilon$ $=0.75$. Indeed, the advantage of the straight cone tooth is only modest when compared with the much blunter $\varepsilon=0.55$ tooth for pig hide. The results for deer and pig hide are broadly similar, but sharper teeth have a greater relative advantage in the deer hide. We wondered whether this difference in the shape of the force curves had anything to do with the deer's thick fur. To test the idea, we removed all the fur from a section of deer hide with a razor and punctured it with both the 0.333 and 1.0 teeth from the $\varepsilon$ series. Fur has a significant effect on both teeth; however, the effect was greater on the sharp tooth (about 29\% more difficult to puncture, d.f. $=13, t=4.33, P<$ 0.002 ) than for the dull tooth (about $17 \%$ harder, d.f $.=13, t=$ $4.07, P<0.002)$. These results are not consistent with differences in data between pig and deer.

The manner of penetration differs based on the sharpness of the teeth in the $\varepsilon$ series. Because of the toughness of the hide of mammals, blunt teeth require a great deal of force for penetration. Initial puncture by blunt teeth is accompanied by a popping sound. After the catastrophic failure of the hide, much less force is needed to complete full penetration (Figure 4). Sharp teeth have a much easier time pricking through the hide initially, but pushing the ever-expanding shank of the tooth requires much more force than the initial prick. The force required to penetrate through deer and pig hide for the aspect ratio series of artificial teeth is shown in Figure 6b. For both hides, our results show that teeth with higher aspect ratios (narrower teeth) penetrate more easily.

\section{Discussion}

One non-intuitive result that we found from puncturing mammal hide was that the sharp cone did not have much of an advantage in puncturing over considerably blunter teeth (Figure $6 a)$. This may have to do with the thickness of the hide and the height of the tooth. At $12 \mathrm{~mm}$, the tooth is much longer than the hide is thick (about $3 \mathrm{~mm}$ ). The sharp point of the conical tooth is excellent at penetration (Figure 4a). Once that sharp tooth passes through the hide, the much softer fat and muscle below offers little resistance compared with the force needed for the shank of the tooth to expand the hole in the hide as the tooth penetrates deeper. The advantage of the cone might be sustained if the hide were thicker than the height of the tooth. This is not the case for most cats and their prey. It should be noted that our standard for measuring force of penetration is based on finding the maximum force needed to push the tooth through the hide to the tooth's base. If initial pricking were all that were required for successful capture of the prey, very sharp pointed teeth would be at a greater advantage than our data indicate (Figure 6a).

Can an optimal $\varepsilon$ be predicted with our data? Thus far, we have concentrated on two curves: tooth shape parameter, e, versus tooth weakness (Figure 5a) and $\varepsilon$ versus the force needed to penetrate a hide (Figure 6a). The weaker a tooth, the higher the probability a tooth will break and this may incur a loss of fitness or cost for the predator. Also, the blunter the tooth, the harder to penetrate the prey's hide, and this might also incur a cost. When we combine these two cost curves, the optimal tooth shape would be the one that sustains the lowest total cost. The trouble is that we have no idea what the costs
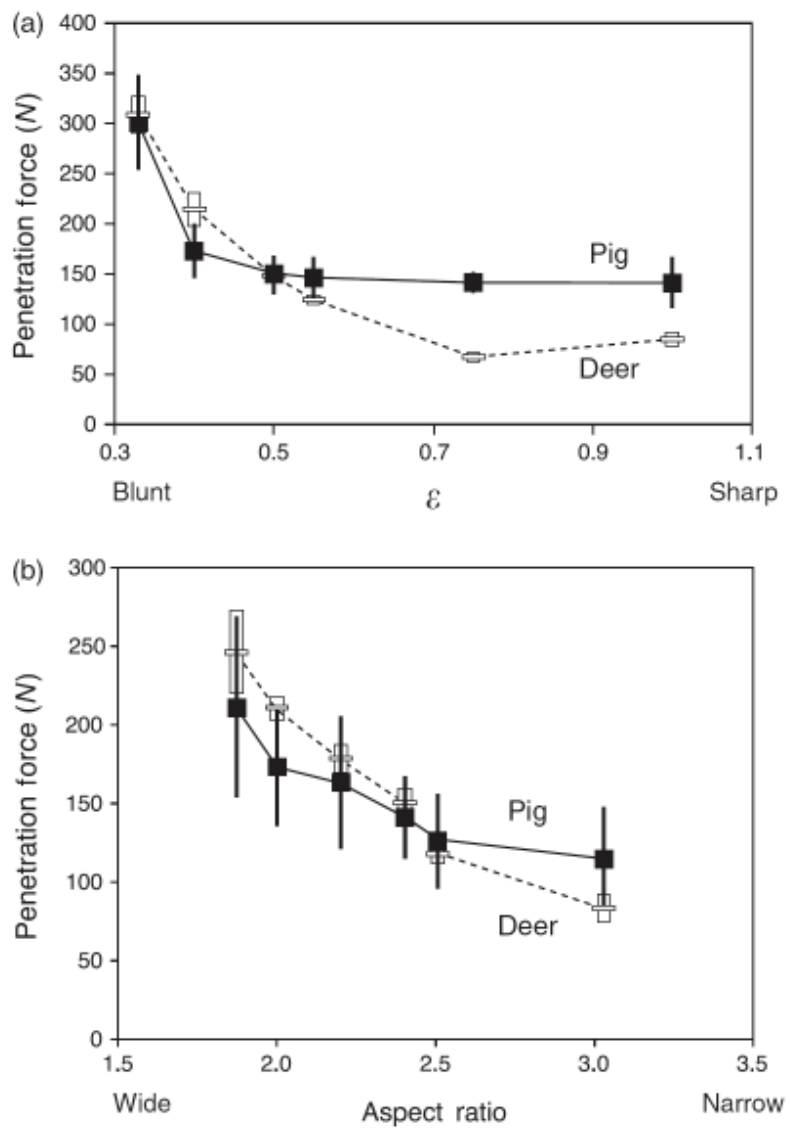

Figure 6 (a) Maximum penetration force needed to penetrate deer Odocoileus virginianus (open squares) and pig Sus scrofa domesticus (solid squares) hide for the taper parameter, $\varepsilon$, series of teeth (aspect ratio $=2.5$ ). Bars indicate $95 \%$ confidence limits on the mean. (b) Maximum penetration force needed to penetrate deer (open squares) and pig (solid squares) hide for the aspect ratio series of teeth $(\varepsilon=0.55)$. Bars indicate $95 \%$ confidence limits on the mean. 
of breakage and failure to penetrate are relative to each other and therefore have no way to add up the costs. However, as a simplifying assumption we can imagine that there is a simple linear combination of cost near the optimal solution:

$$
\text { Costs }=\alpha W+(1 / \alpha) P
$$

where $W$ is the weakness of a tooth and $P$ is the force needed to penetrate a hide. Alpha alters the relative importance of $W$ and $P$ in the calculation of costs. Once again, we do not know what the real $\alpha$ is, and so we cannot plug it into this equation. Unlike $\varepsilon$, which is an absolute measure based on standardized tooth shape, $\alpha$ is contextual. When combining the force of penetration and weakness to form a cost [equation (2)], the force will be dependent on the hide used and the type of weakness at the tip (e.g. 5 or $20 \%$ tip weakness). With this in mind, we varied a over a wide range, from 0.1 to 10 , and calculated the costs using deer hide at $5 \%$ tooth weakness (Figure 7). This means that the relative weight of $P$ and $W$ in equation (2) varies from 100:1 to $1: 100$ in this figure. When penetration is 100 times more important than strength $(\alpha=0.1)$, the sharp teeth (higher $\varepsilon$ 's) have the lowest costs and should be the optimum solution (Figure 7). Conversely, when strength is 100 times more important, the blunt tooth (lower $\varepsilon$ ) is the optimal choice. As no felids use either extreme tooth form, we expect that the true value of $\alpha$ lies between 0.1 and 10 .

Unfortunately, we are not able to predict the optimal $\varepsilon$ based on our experimental data because we lack knowledge of $\alpha$. Indeed, $\alpha$ is likely a very difficult quantity to measure. However, $\varepsilon$ can be measured easily. The values for several felids are shown in Table 2. Therefore, instead of predicting $\varepsilon$ based on a, we use $P, W$, and $\varepsilon$ to predict $\alpha$. There is a drawback to this approach; we will not be able to check our predictions of a against experimentally determined values that would have been possible if we could have predicted the taper parameter $\varepsilon$. However, as discussed below, armed with experimentally determined $\varepsilon, P$ and $W$ and an inferred $\alpha$, we will be able to predict optimal aspect ratios, values that can be compared with aspect ratios for felids.

Measuring several felids, we found values of $\varepsilon$ to be conservative and around $0.5-0.55$ (Table 2). Using our $W$ and $P$ data from pig and deer hide, we can ask what values of $\alpha$ would result in a tooth with $\varepsilon$ at about $0.5-0.55$ being the optimal solution. In Figure 7, we have drawn a horizontal line at $\alpha=2$. In Figure 8, we plot the costs along this line and note that the lowest cost occurs at $\varepsilon=0.55$ for this value of $\alpha$. Based on this approach, we found the range of $\alpha$ values for which the optimal solution for $\varepsilon$ was $0.5-0.55$. This range of a values was determined for all four cases: deer 5\% tip weakness, deer 20\% tip weakness, pig 5\% tip weakness and pig $20 \%$ tip weakness.

While interesting in their own right, $\alpha$ values can be used to predict the optimal aspect ratios of felid teeth. First, we followed the same method described above to calculate the costs in the $\varepsilon$ series teeth to now calculate the costs for the aspect ratio tooth series. As before, we calculated costs over the range of a from 0.1 to 10 . Then, we determined the optimal aspect ratio (lowest cost) for each of these $\alpha$ values and plotted the results in Figure $9 \mathrm{a}-\mathrm{d}$ for both pig and deer and 5 and $20 \%$ tip weakness. When strength is relatively unimportant, the optimal tooth will have a high aspect ratio (Figure 9).

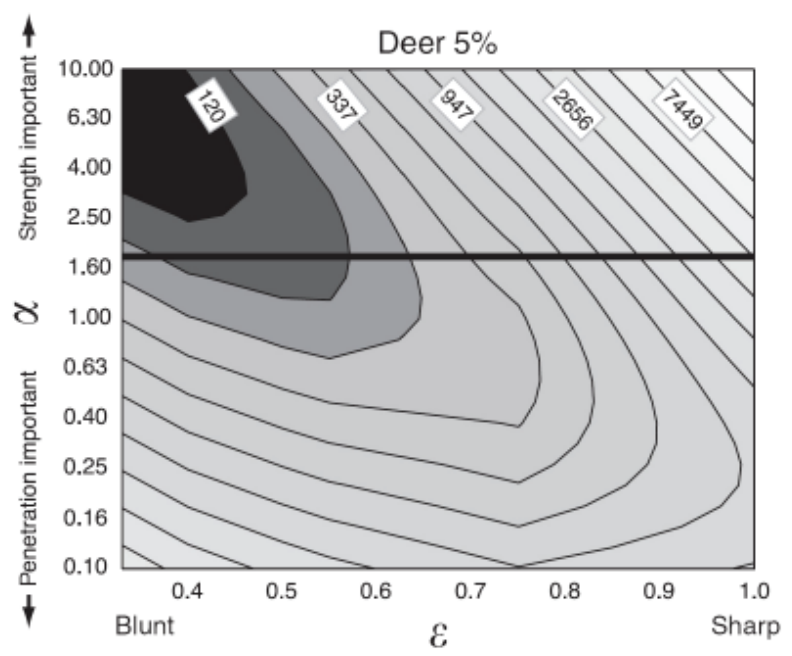

Figure 7 Impact of $\varepsilon$ on costs (combination of weakness and force of penetration) over a wide range of the relative weight factor for weakness and penetration, $\alpha$. Darker shading represents lower costs. At the upper left of the graph, $\alpha$ is 10 ; hence, weakness is relatively important and the bluntest tooth $(\varepsilon=0.333)$ is favored. Toward the lower right of the graph, $\alpha$ is 0.1 , and force of penetration is relatively important and sharper teeth are favored $(\varepsilon=0.75)$. Costs along the line drawn at $\alpha=$ 2 are plotted in Figure 8.

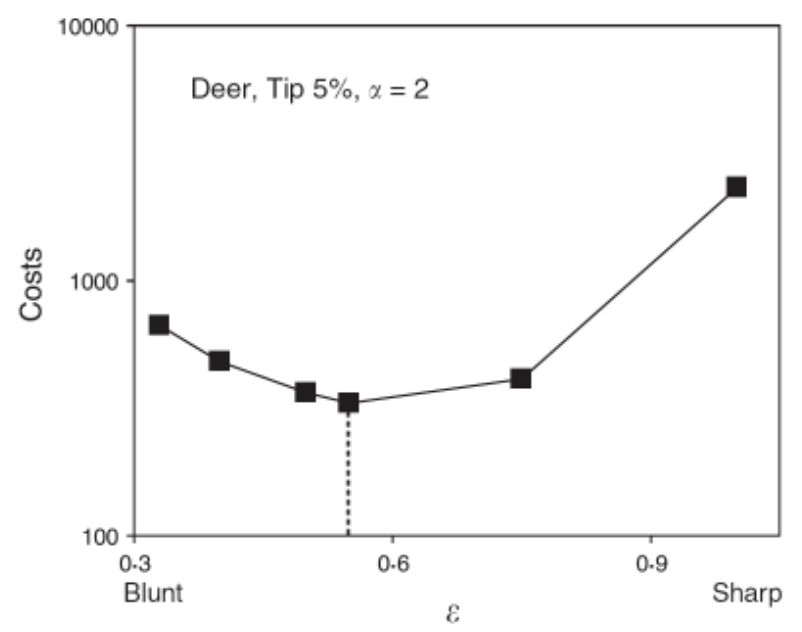

Figure 8 Costs plotted for the $\varepsilon$ series of teeth with $\alpha=2$. Graphically, these are the costs along the horizontal line plotted in Figure 7. Note that the lowest cost, and hence the optimal $\varepsilon$ for this $\alpha$ is 0.55 (dashed line). 
(a)

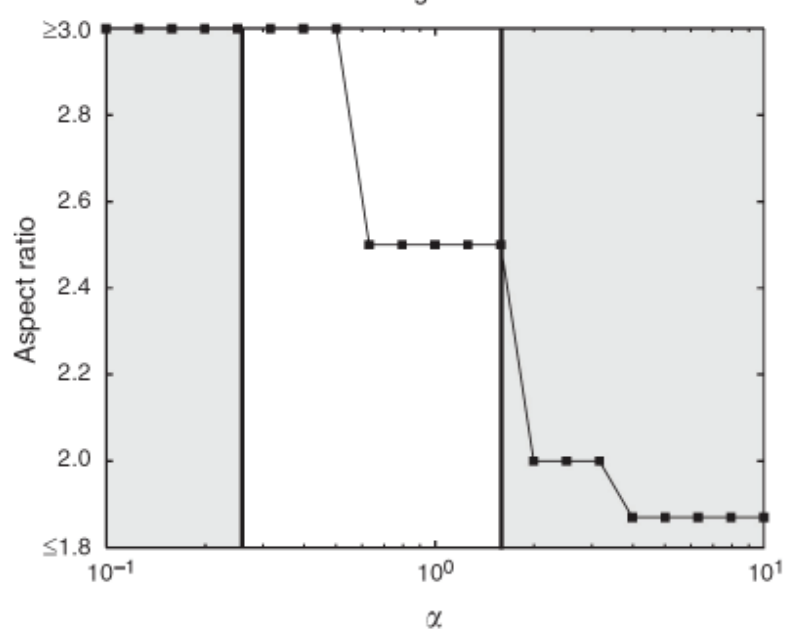

(c)

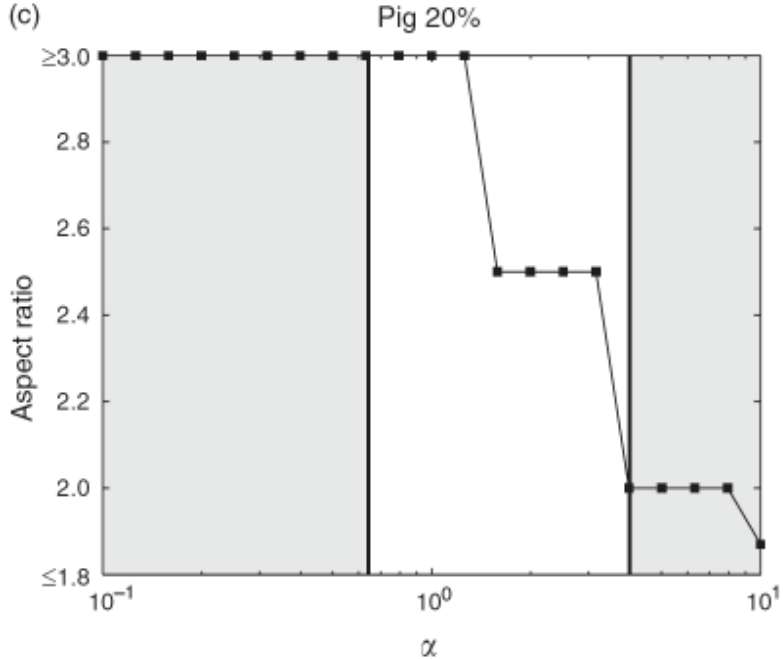

(b)

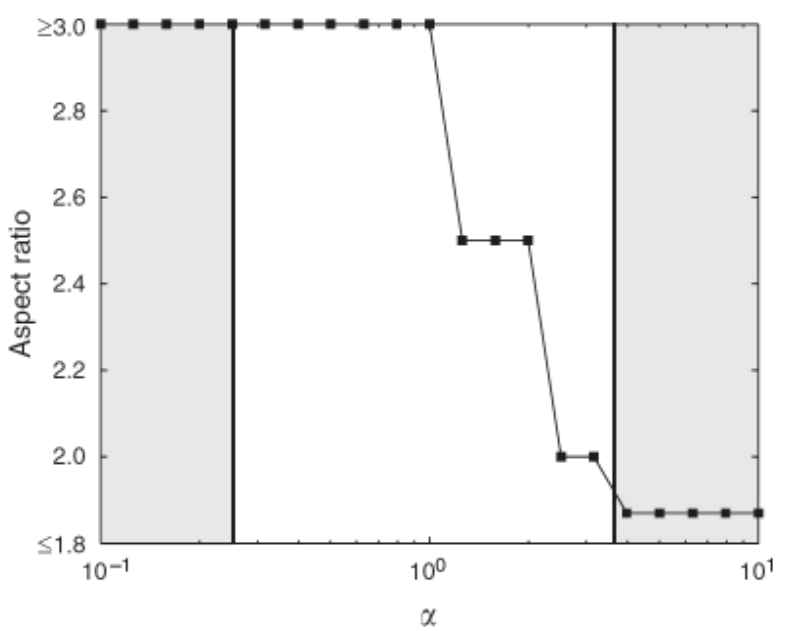

(d)

Deer $20 \%$

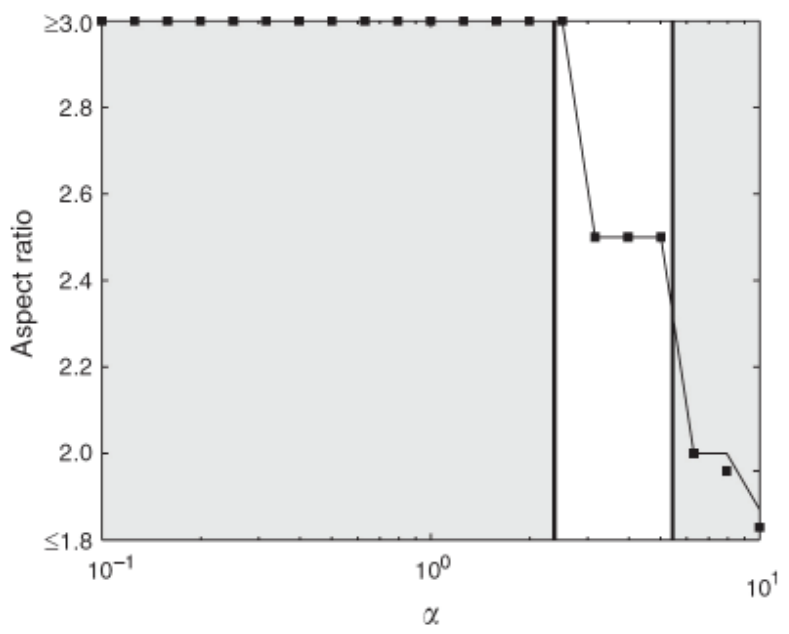

Figure 9 In this series of plots, the connected squares represent the optimal aspect ratios for different values of a for pig Sus scrofa domesticus $5 \%$ (a), pig $20 \%$ (b), deer Odocoileus virginianus 5\% (c) and deer $20 \%$ (d). On the left, $\alpha$ is low and weakness has a relatively low cost, but force of penetration has a high cost; hence, a high aspect ratio is optimal. The opposite is true on the right, which is a condition that favors low aspect ratios. The vertical lines in each plot represent the range of $\alpha$ 's for which the optimal $\varepsilon$ value is from 0.5 to 0.55 . The unshaded area between the vertical lines represents our predictions of optimal aspect ratios.

Note that in our artificial tooth series an aspect ratio of 3.0 is the highest made, and so our model cannot predict even higher aspect ratios as optimal. As a result, we have labeled this as $\geq$ 3.0 on the graph to indicate that an aspect ratio of at least 3.0 is optimal. Similarly, our lowest aspect ratio is 1.8 , which we have labeled as $\leq 1.8$ to indicate that an aspect ratio of this value or lower is optimum. Figure 9 shows that a very wide range of aspect ratios can be predicted based on a values varying from 0.1 to 1 . However, in our experiments on the $\varepsilon$ series of teeth we narrowed the range of expected $\alpha$ values to those that predicted $\varepsilon$ values of $0.5-0.55$ (values found in felids). These ranges are shown in Figure 9 as vertical lines superimposed on the graphs. For deer hide and pig hide and the 5 and $20 \%$ tip data, the predicted aspect ratio ranged between 3 and
2 and centered on 2.5. Aspect ratios of a variety of wild felids are shown in Table 1 and have a range of 2.1-3.3 with an average of 2.39. Thus, there is strong agreement between our predicted optimal aspect ratios and those found in wild cats.

However, there are also exceptions, some quite spectacular, of canines of species not included in Table 2. Felids sometimes have very long teeth (saber-toothed cats from the fossil record). It is not clear what the advantage is for these long, ovoid canines and considerable controversy exists for the role of saber teeth (Emerson \& Radinsky, 1980). The failure of our predictions to match all cats could be caused by differences in the cost of breakage or differences in hide toughness. Finally, the tooth's morphology could be controlled by factors beyond strength and force of penetration. 


\section{Acknowledgements}

We thank the School of Natural Resources for their support, the Division of Zoology, University of Nebraska State Museum for the use of specimens in their collection and Angie Fox, Museum Artist, for assistance with figures.

\section{References}

Alexander, R.McN. (1982). Optimum structures - tubular bones. In Optima for animals: 13-17. Alexander, R.McN. (Ed.). London, UK: Edward Arnold Ltd.

Emerson, S.B. \& Radinsky, L. (1980). Functional analysis of sabertooth cranial morphology. Paleobiology 6, 295312.
Evans, A.R. \& Sanson, G.D. (1998). The effect of tooth sharpness on the breakdown of insects. J. Zool. (Lond.) 246, 391-400.

Popov, E.P. (1999). Engineering mechanics of solids. New Jersey: Prentice-Hall.

Van Valkenburgh, B. (1988). Incidence of tooth breakage among large, predatory mammals. Am. Nat. 131, 291302.

Van Valkenburgh, B. \& Ruff, C.B. (1987). Canine tooth strength and killing behaviour in large carnivores. $J$. Zool. (Lond.) 212, 379-397.

Weibel, E.R. (2000). Symmorphosis: on form and function in shaping life. Cambridge, USA: Harvard University Press. 